\title{
Post-Denosumab-Treated Giant Cell Tumor of Bone: A Retrospective Histomorphological and Immunohistochemical Study
}

\author{
Anvesh Kamble ${ }^{1}$ Monalisa Hui ${ }^{1}$ \\ G. Sadashivudu ${ }^{4}$ \\ 1Department of Pathology, Nizam's Institute of Medical Sciences, \\ Hyderabad, Telangana, India \\ 2Department of Orthopaedics, Nizam's Institute of Medical \\ Sciences, Hyderabad, Telangana, India \\ ${ }^{3}$ Department of Radiology and Imaging, Nizam's Institute of \\ Medical Sciences, Hyderabad, Telangana, India \\ ${ }^{4}$ Department of Medical Oncology, Nizam's Institute of Medical \\ Sciences, Hyderabad, Telangana, India
}

Ind J Med Paediatr Oncol 2021;42:325-332.
N. Ramakrishna ${ }^{3}$ P. Chandrasekhar ${ }^{2}$

\author{
Address for correspondence Shantveer G. Uppin, MD, Department \\ of Pathology, Nizam's Institute of Medical Sciences, Punjagutta, \\ Hyderabad, Telangana 500082, India \\ (e-mail: drsguppin@yahoo.co.in).
}

\section{Abstract}

Keywords

- giant cells

- denosumab therapy

- histomorphological changes

- antihistone antibody

- immunohistochemistry
Introduction Giant cell tumors of bone (GCTBs) are treated with surgery with or without local adjuvants. Denosumab is a human monoclonal antibody that has recently emerged to be effective in treating unresectable and recurrent GCTBs.

Objective In this study, we analyzed the histomorphological changes in GCTB following treatment with denosumab. The expression of histone mutation $\mathrm{H3} .3 \mathrm{G} 34 \mathrm{~W}$ by immunohistochemistry (IHC) using mutant specific antibody was also determined. Materials and Methods Of the total 109 GCTBs encountered during the study period, 14 cases with neoadjuvant denosumab therapy were analyzed retrospectively. The post-treatment changes on histopathology were examined on routine hematoxylin and eosin-stained sections. IHC was done using antihistone H3.3G34 antibodies. Statistical analysis was limited to descriptive statistics. No hypothesis testing was performed.

Results All these cases except three showed fibrosis with areas of hyalinization, prominent newly formed woven bone along with spindle cells in short fascicles and storiform pattern. There was complete absence and marked reduction in osteoclast-like giant cells in six and five patients, respectively. Only three patients showed a substantial amount of residual osteoclast-like giant cells. IHC with antihistone $\mathrm{H3} .3 \mathrm{G} 34 \mathrm{~W}$ antibody showed unequivocal nuclear positivity in the mononuclear cells in nine cases. The mononuclear cells rimming and entrapped within the woven bone were also positive on IHC. The spindle cells in the benign fibrous histiocytoma-like areas and septa of aneurysmal bone cyst-like areas also retained nuclear staining.

Conclusion Awareness of post-denosumab-related histopathological changes are necessary to avoid misdiagnosis as fibroosseous lesion and low-grade central osteosarcoma. Expression of mutant-specific H3.3 G34W antibody suggests that the neoplastic stromal cells are largely retained after denosumab therapy. The positive staining of cells both within and those rimming the newly formed woven bone point toward osteoblastic phenotype of the neoplastic stromal cells. (c) 2021. Indian Society of Medical and Paediatric Oncology.

This is an open access article published by Thieme under the terms of the Creative Commons Attribution-NonDerivative-NonCommercial-License, permitting copying and reproduction so long as the original work is given appropriate credit. Contents may not be used for commercial purposes, or adapted, remixed, transformed or built upon. (https://creativecommons.org/licenses/by-nc-nd/4.0/).

Thieme Medical and Scientific Publishers Private Ltd. A-12, Second

Floor, Sector -2, NOIDA -201301, India 


\section{Introduction}

Giant cell tumor of bone (GCTB) is a locally aggressive neoplasm composed of mononuclear neoplastic cells with an osteoblastic precursor phenotype, mononuclear macrophage, and osteoclast-like multinucleated giant cells. These typically affect ends of long bones and are generally treated with surgery, either en-bloc resection or curettage with or without local adjuvants. The mononuclear neoplastic cells in GCTB express high levels of receptor activator of nuclear factor NF-K $\beta$ ligand (RANKL) that binds to tumor necrosis factor-induced receptor activator of nuclear factor NF-K $\beta$ (RANK) receptors expressed on multinucleated osteoclast-like giant cells and its mononuclear precursors. ${ }^{1}$ Such interaction of RANK-RANKL leads to recruitment of osteoclast precursors promoting osteoclastogenesis and bone resorption. The RANKL/RANK/OPG system plays an important role in the regulation of bone growth and turnover. Osteoprotegerin (OPG) acts as a decoy receptor for RANKL and inhibits the stimulation of osteoclast differentiation. In normal physiological conditions, there is a balance between RANK, RANKL, and OPG. ${ }^{2,3}$

Denosumab is a human monoclonal antibody that binds to RANKL with high affinity, preventing its interaction with RANK on the surface of osteoclasts and its precursors. ${ }^{4,5}$ Studies have shown its efficacy in treating unresectable GCTB as well as in neoadjuvant settings. In this study, we have analyzed the histomorphological changes in GCTB following treatment with denosumab. Though literature on histopathology of post-denosumab-treated GCTBs has been published worldwide, very few studies have been reported from India. ${ }^{4,6-8}$ Moreover, immunohistochemistry (IHC) with antihistone H3.3G34W antibodies, a novel diagnostic marker of GCTB has not been widely performed in this subset of cases. In this article, apart from assessing the histomorphological changes, we also attempted to determine the expression of histone mutation H3.3G34W by using mutant specific antibody.

\section{Materials and Methods}

Of the total 109 cases of GCTB encountered during the study period (2018-2020), fourteen (14) cases of Campanacci grade III GCTB who had received neoadjuvant denosumab treatment were analyzed retrospectively to study post-treatment changes. The remaining 95 patients who did not receive denosumab following a histological diagnosis of GCTB were excluded from the study. The demographic data, location of the tumors, imaging findings, and treatment history were retrieved from the medical records. The initial diagnosis of GCTB was made on core needle biopsy (4 patients), curettage specimen ( 6 patients), and fine needle aspiration smears along with cell block (4 patients). All the cases except one were initially diagnosed outside and referred to our institute for further management. Following confirmation of diagnosis, all the patients received three cycles of $120 \mathrm{mg}$ denosumab subcutaneously on day 0 , day 8 , and day 15 . Patients were followed up for 1 month and repeat radiographs were taken prior to surgery to assess the effectiveness of denosumab therapy. After formation of a bony shell around the tumor on imaging, surgery was done that included resection in eight patients and curettage followed by bone grafting in six patients. The curettage specimens were submitted for histological examination entirely. Representative tumor sections were examined from the excised specimens. The sections were processed for routine paraffin sections after fixation in $10 \%$ buffered formalin. Decalcification was performed with $10 \%$ nitric acid whenever necessary prior to processing. The histopathological features on routine hematoxylin and eosin-stained sections were analyzed in all the cases. The representative formalin fixed paraffin embedded tissue blocks were chosen for IHC. An attempt was made to exclude the sections subjected to prior decalcification wherever possible and care was taken not to include the hemorrhagic and necrotic areas. In five cases, nondecalcified sections were not available, so IHC was performed on decalcified tissue sections.

IHC was performed using antihistone rabbit monoclonal antibody H3.3G34W (clone RM 263, Medasys Livermore, California, United States; dilution 1:100) on Roche Ventana Benchmark GX fully automated platform. The presence of unequivocal crisp nuclear staining with H3.3G34W in the cells irrespective of intensity and proportion was considered as positive staining. A case of GCTB with classic histomorphology and positive staining on IHC was taken as a positive control.

Postoperative recovery was uneventful in all the cases. None of them received any adjuvant denosumab following surgery. Follow-up details were documented wherever available. Clinical symptoms after denosumab treatment and last follow-up after surgery were noted.

\section{Statistical Analysis}

Statistical analysis was limited to descriptive statistics using Microsoft Office Excel 2016, Singapore. No hypothesis testing was performed.

\section{Ethics}

The study was approved by Nims Institutional Ethics Committee (EC/NIMS/2338/2019, dated 22.03.2019) after obtaining waiver of informed consent. The procedures followed were in accordance with the ethical standards of the responsible committee on human experimentation (institutional) and with the Helsinki Declaration of 1964, as revised in 2013.

\section{Results}

Of the total 14 patients studied, there were equal number of males and females. The age of the patients ranged from 17 to 47 years with a mean of 25.8 years. All the patients presented with pain and swelling of the affected sites. Pathological fracture was noted in one patient. Of the 14 patients, 11 had presented with primary tumor and remaining 3 were recurrent. The tumors recurred following initial curettage after a period of 8 months, 2 years, and 4 years. All except four patients 
showed resolution of the clinical symptoms (pain and swelling) to a large extent.

Initial (pre-treatment) plain radiographs showed expansile lytic lesion in the epiphysis ( $\boldsymbol{- F i g . ~ 1 A}$ and $\mathbf{B}$ ). Magnetic resonance imaging done in 12 patients had revealed an additional component of aneurysmal bone cyst $(A B C)$ in three patients. Post-treatment radiographs taken after three doses of denosumab showed varied reduction in tumor size, marginal sclerosis, and internal ossification ( - Fig. 1C and D ).

On histopathology, all these cases except three showed prominent newly formed woven bone along with spindle cells arranged in short fascicles and storiform pattern. Areas of fibrosis and hyalinization were noted. The other changes included focal necrosis (5 cases), collection of foamy macrophages ( 7 cases), inflammatory infiltrate ( 3 cases), cholesterol clefts ( 1 case), and foreign body giant cells (1 case). The inflammatory cells chiefly comprised of lymphocytes and plasma cells admixed with few neutrophils. Some of the cases showed prominent ectatic thin walled vessels. ABC-like changes were noted in two patients and benign fibrous histiocytoma (BFH)-like area was seen in one patient. There was marked reduction in osteoclast-like giant cells in five patients, complete absence in six patients, and remaining three patients showed substantial amount of residual osteoclast-like giant cells (-Fig. 2). The location, imaging findings, and histopathological changes in post-denosumab-treated GCTB are summarized in - Table 1.

IHC with antihistone H3.3G34W antibody done in all the nine cases showed unequivocal nuclear positivity. The intensity of the staining was strong to moderate and was restricted to the mononuclear cells. None of the residual osteoclast-like giant cells were positive on immunostaining. The mononuclear cells rimming and entrapped within the woven bone were also positive on IHC ( - Fig. 3 ). In addition, staining was retained in some of the spindle cells in the BFH-like areas and septa of $\mathrm{ABC}$-like areas. In one case, the original tumor (pre-treatment biopsy sample) tested also showed similar positivity.

There were five cases where IHC with antihistone antibody was attempted on decalcified sections. The IHC results were negative in all these five cases that can be attributed to decalcified tissue sections. In these cases, pre-treatment tissue samples were not available for testing as these were referral cases diagnosed outside.

On follow-up, one patient developed recurrence after a period of 2 years following surgery. This patient presented with a fungating mass in the right proximal tibia and above knee amputation was done. Histopathological sections revealed a residual tumor along with denosumab-related changes including fibrosis, hyalinization, newly formed woven bone, and focal BFH-like areas. Post-surgery, follow-up in another 9 patients showed no evidence of disease after a period of 2, 3, 13, 14, and 24 months. Four patients who were lost to follow-up at $12,15,18$, and 24 months had not shown any evidence of recurrence at their last visit.

\section{Discussion}

Denosumab has emerged as a new treatment option for GCTB and is indicated especially in large unresectable lesions, recurrent tumors, and metastasis. Denosumab is a RANKL inhibitor preventing its interaction with RANK and inhibits bone destruction. ${ }^{910}$ It shifts the dynamics of bone formation away from RANK-mediated osteoclastic bone resorption toward osteoprotegerin-induced bone formation. The mononuclear stromal cells that are the true neoplastic cells in GCTB differentiate to osteoblastic phenotype, leading to deposition of newly formed osteoid/ woven bone after treatment with denosumab. ${ }^{5}$ Thus, extensive newly formed osteoid/woven bone deposited in post-denosumab-treated cases is produced by the neoplastic stromal cells of GCTB.
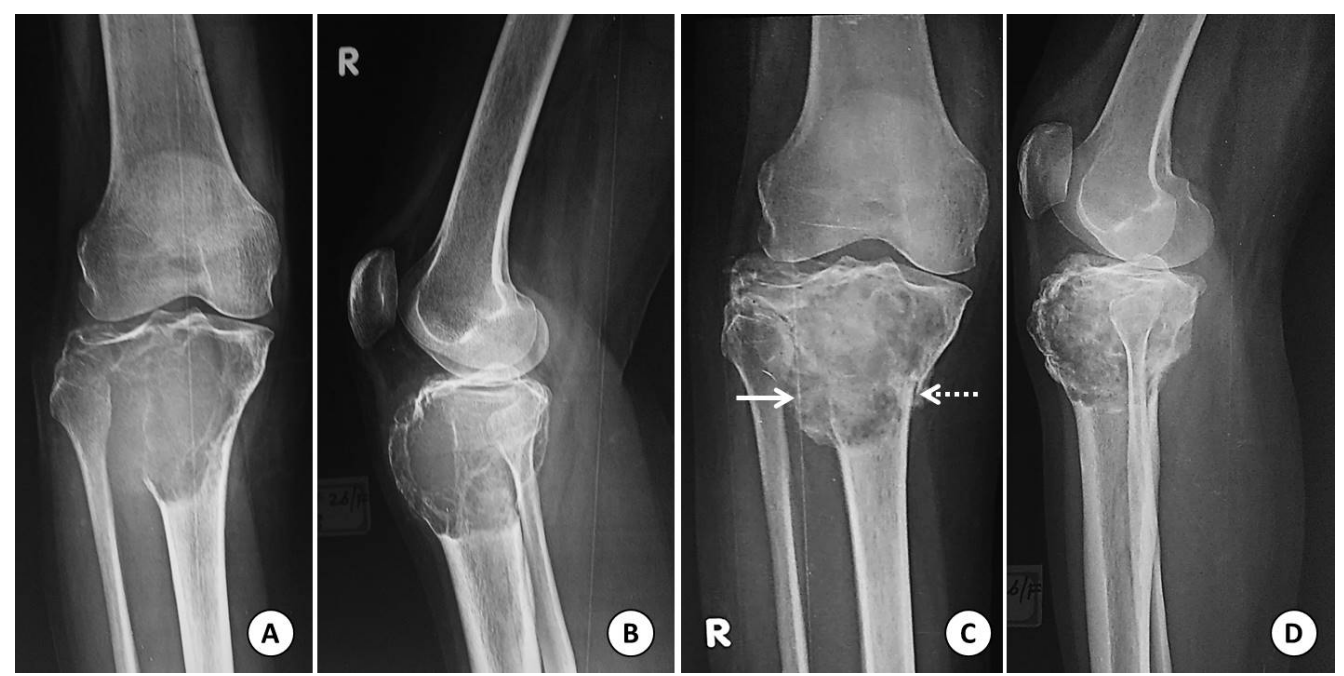

Fig. 1 (A and B) Pre-treatment radiographs of giant cell tumor of bone involving right proximal tibia. (A) Anteroposterior and (B) lateral radiographs showing a large geographic mildly expansile locally aggressive lytic lesion in proximal tibia with ambiguous lateral margins and fine internal septations. (C and D) Post-denosumab treatment radiographs of the same case. (C) Anteroposterior and (D) lateral radiographs showing development of marginal sclerosis (white arrows) and internal ossification. Note there is a small pathological fracture along the medial tibial cortex (dashed arrow). 

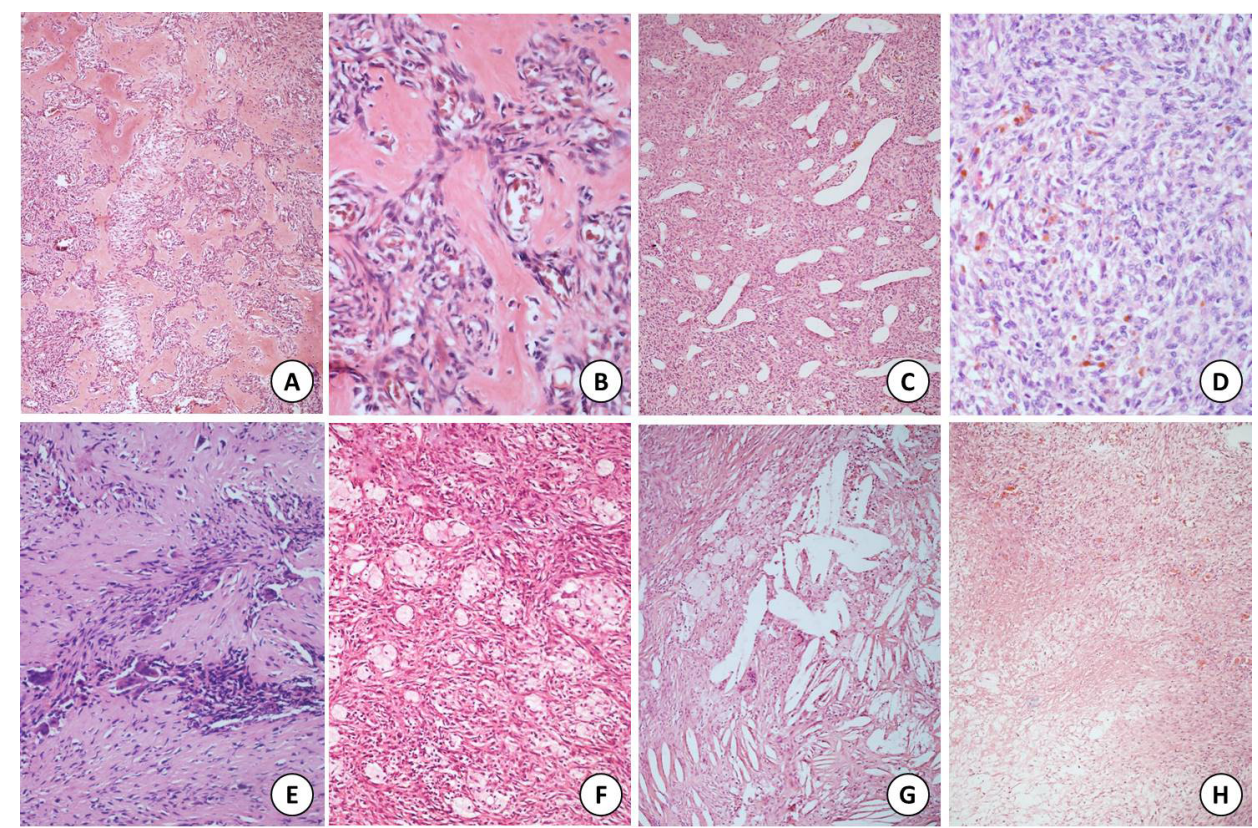

Fig. 2 Hematoxylin and eosin-stained histological sections of post-denosumab-treated giant cell tumor of bone. (A) Lower magnification $[\times 100]$ and $(B)$ higher magnification image showing prominent interconnecting network of newly formed woven bone with intervening mononuclear spindle cells [×400]. (C) Prominent ectatic thin-walled vessels [×100]. (D) Prominent mononuclear oval to spindle cells. Note few focally admixed hemosiderin laden macrophages $[\times 200]$. (E) Areas of fibrosis with entrapped spindle cells and few residual osteoclast-like giant cells [ $\times 200$ ]. (F) Benign fibrous histiocytoma-like focus showing storiform arrangement of spindle cells with admixed foamy macrophages [ $\times 100]$. (G) Focus showing prominent cholesterol clefts $[\times 100]$. (H) Area of necrosis $[\times 100]$.

Denosumab reduces tumor volume and leads to formation of a rim of ossified bone at the periphery. This facilitates the process of curettage and minimizes the risk of injury to the neurovascular structures lying in close proximity to the lesion. The current guidelines recommend the use of neoadjuvant denosumab for a period of 3 to 4 months. Prolonged use may be indicated only in surgically unsalvageable GCTB. ${ }^{11}$ In our setup, all the patients underwent definite surgical procedure after a period of 1 month only.

Deveci et al graded all tumors after surgery according to a two-tier grading system. Grade I tumors are composed of densely cellular mononuclear cells and osteoclast-like giant cells. The grade II tumors indicate transition to osteoblastic lineage showing marked woven bone formation, osteoid, focal mononuclear cells, and markedly reduced osteoclast-like giant cells. ${ }^{4,12}$ Most of the cases in the present study except three (Case 9, 11, and 12) were nonproliferative grade II tumors with marked woven bone formation. Similar histopathological changes were noted in other studies as well. $6,7,12,13$

As currently denosumab is frequently administered to treat GCTB, it is important to be familiar with the various morphological changes it induces, because there is a considerable overlap between these and primary tumors of the bone. The histologic changes in denosumab-treated GCTBs mimick fibroosseous lesions of bone (fibrous dysplasia/nonossifying fibroma), low-grade central osteosarcoma, and osteoblastoma. Presence of prominent woven bone with spindle cells showing mild nuclear atypia strongly resembles low-grade central osteosarcoma, leading to erroneous diagnosis. Most of the cases in the present study mimicked fibroosseous lesion of the bone. Hence, pathologist dealing with such specimens should be aware of these histopathological findings and should seek prior treatment history if such changes are encountered in cases with a clinical and imagological diagnosis of GCTB to avoid misdiagnosis.

Wojcik et al reported marked reduction in osteoclast-like giant cells in all the nine cases analyzed. ${ }^{6}$ There was marked reduction/absence of giant cells along with a fibroosseous component in 11 out of 14 tumors in the present study. Similar findings have been observed in other studies as well. ${ }^{13,14}$

Majority of GCTBs harbor H3.3G34W mutation, in which few of them have alternate mutation like G34L, G34M, G34R, or G34V substitution. Kato et al and Wu and Hsieh et al detected H3F3A mutation on polymerase chain reaction and genetic sequencing. ${ }^{15,16}$ Behjati et al noted H3F3A mutation only in RUNX2 positive stromal cells sparing CD51+/CD61+ osteoclasts or in $\mathrm{CD} 14+$ osteoclast precursors highlighting osteoblastic properties of the neoplastic cells that are retained in denosumab therapy. ${ }^{17}$ Kato et al performed IHC in nine cases of post-denosumab GCTB and found nuclear positivity in all the cases. ${ }^{15}$ Similar extent and intensity of staining were observed in other studies as well. ${ }^{18} \mathrm{Ud}$ Din et al analyzed 19 cases of pre- and post-denosumab-treated GCTB and found anti-H3.3G34W positivity on IHC in 68.8 and $26.9 \%$ stromal cells, respectively. The authors attributed reduced antigenicity of the neoplastic mononuclear cells as the reason behind reduced expression of the antibody in post-treated GCTBs. There was no significant difference in SATB2 expression of the tumor cells in both the specimens reflecting proosteoblastic properties. ${ }^{14}$ In the present study, 
Post-Denosumab-Treated Giant Cell Tumor of Bone Kamble et al. 329

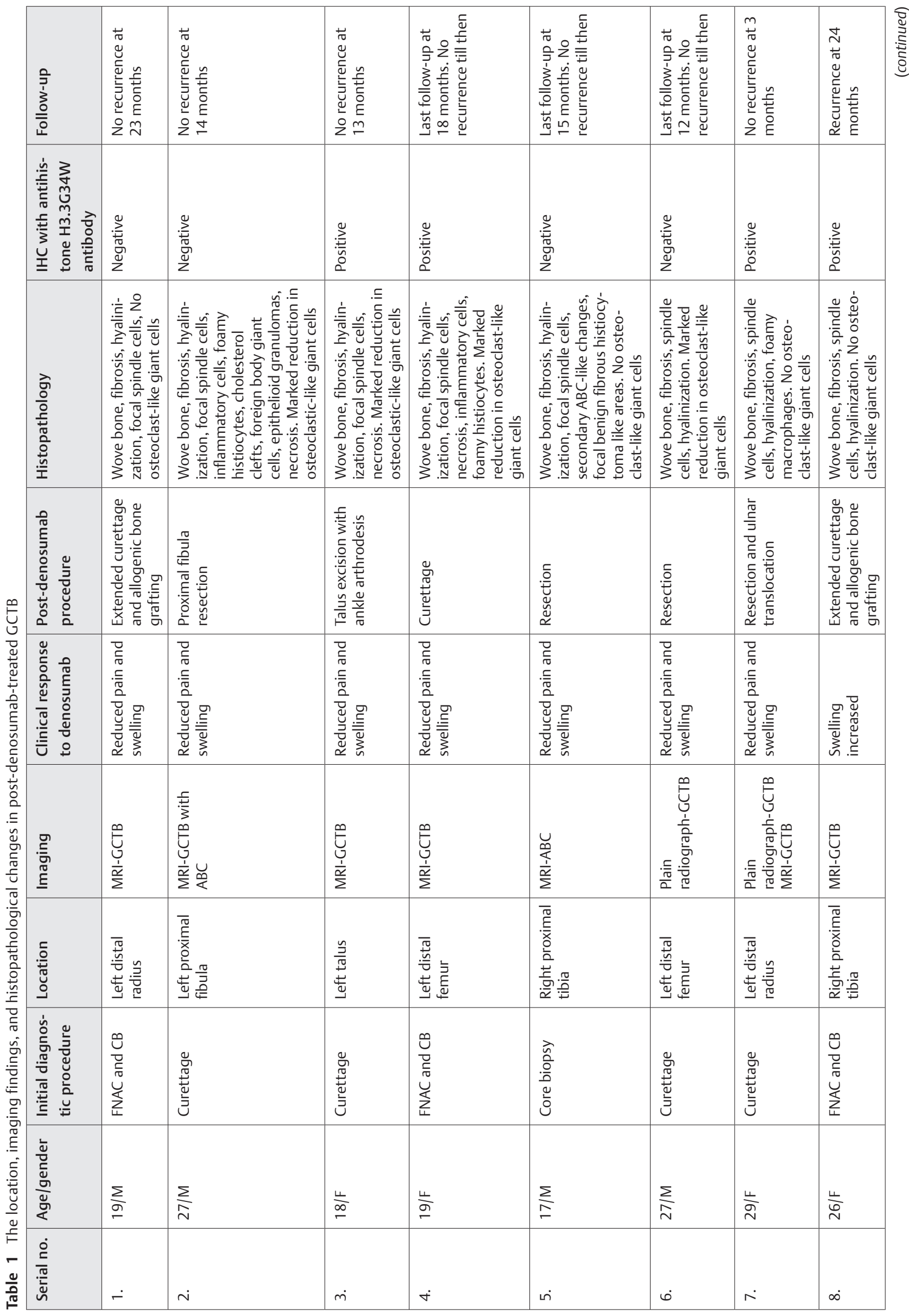


330 Post-Denosumab-Treated Giant Cell Tumor of Bone Ramble et al.

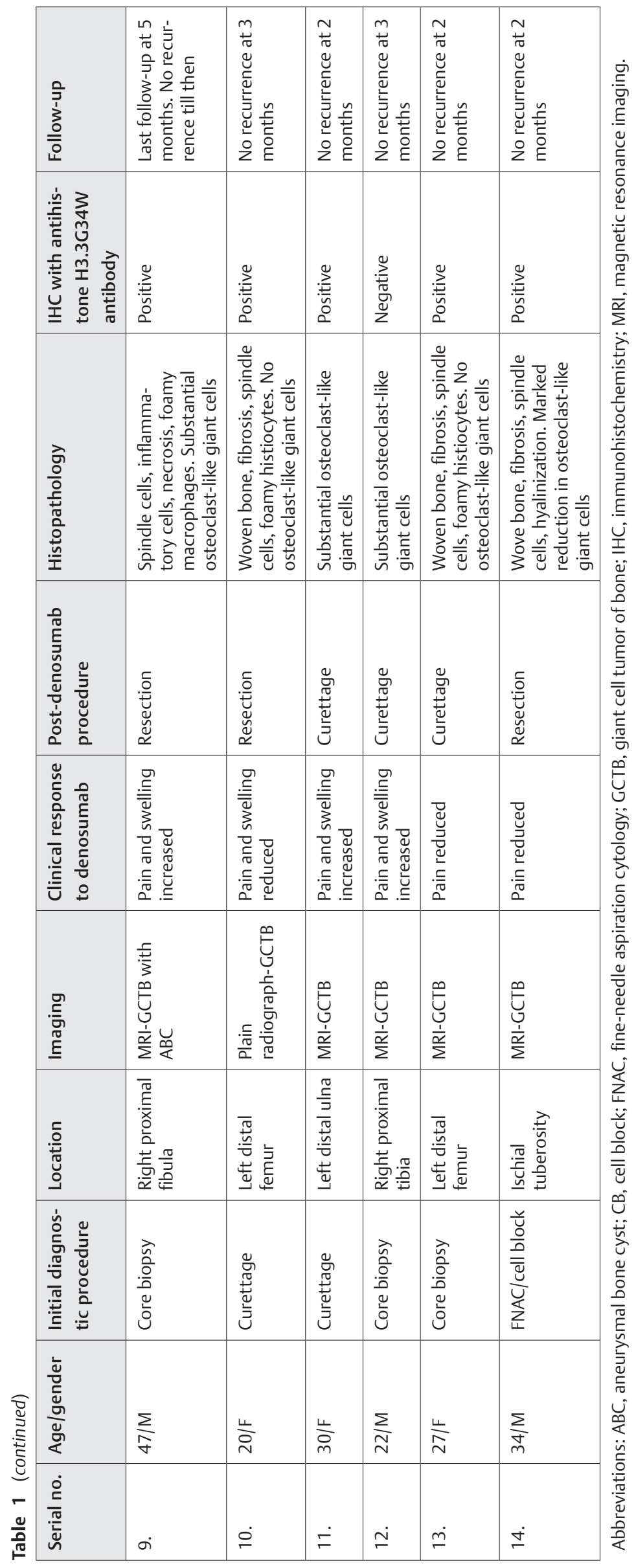

Indian Journal of Medical and Paediatric Oncology Vol. 42 No. 4/2021 $\quad$ @ 2021. Indian Society of Medical and Paediatric Oncology. 


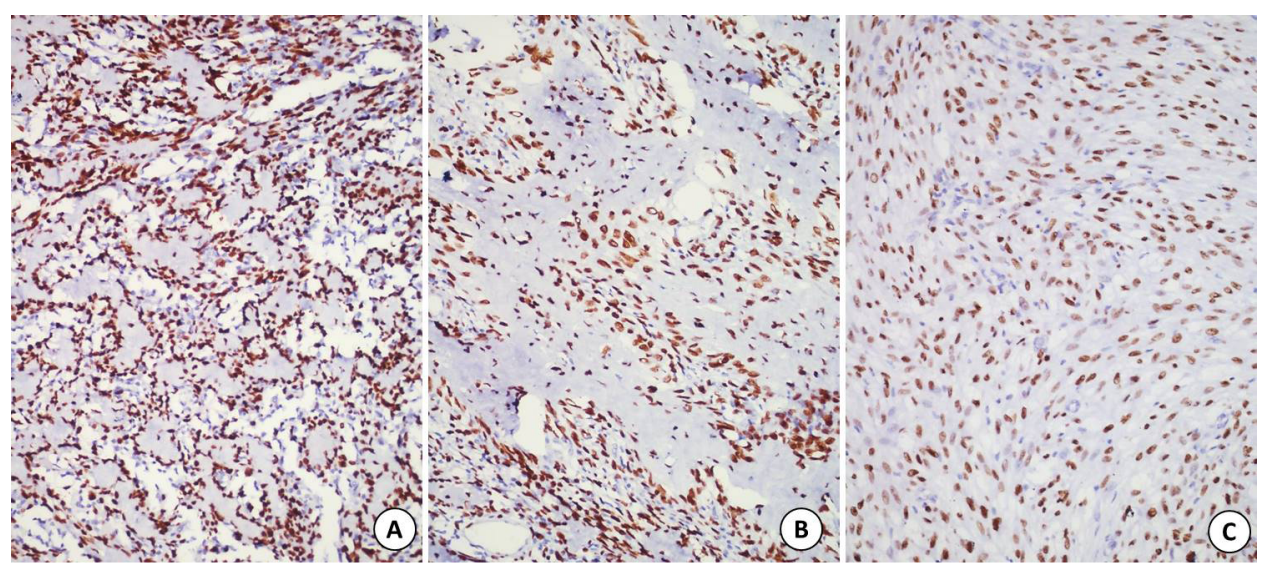

Fig. 3 Immunohistochemistry with antihistone H3.3G34W. (A) and (B) Diffuse strong nuclear staining in the mononuclear cells both lying in between and surrounding prominent network of newly laid woven bone. Strong nuclear staining can also be appreciated in cells embedded within woven bone [A x100, B x200]. (C) Diffuse strong nuclear staining in spindle cell areas devoid of osteoclast-like giant cells [×200].

the pre-treatment biopsy was available in only one case. Hence, a comparative analysis was not studied. SATB2 IHC was not done in the present study. The expression of antihistone antibody in the cells along the bone also indicates maturation toward osteoblastic phenotype of the tumor cells.

Lüke et al found negative expression of antihistone antibody in the cells adjacent to the trabeculae. ${ }^{19}$ In contrast, in our cases positive staining was noted in cells both within and adjacent to bony trabeculae. The five negative cases in the present study were not subjected to sequencing for $\mathrm{G} 34 \mathrm{~W}$ or alternate mutations. In another study, where we have analyzed the expression of H3.3G34 antibody in 135 cases of GCTB (with no prior neoadjuvant denosumab treatment), a positivity $87.3 \%$ was noted (the data are yet to be published). However, in all these cases, IHC was performed on sections of tissue not subjected to prior decalcification process. In the present study, the sections from the resected specimens had all been subjected to prior decalcification with $10 \% \mathrm{HNO}_{3}$. This might be the possible reason for five negative cases in the present study. Further prospective studies are required to see the effect of using various decalcifying agents on IHC results with this antibody.

\section{Conclusion}

Awareness of denosumab-induced histopathological changes in GCTB is essential to avoid misdiagnosing these as fibroosseous lesion or low-grade central osteosarcoma. The demonstration of mutant-specific H3.3 G34W within post-therapy specimen suggest that the neoplastic stromal mononuclear cells of GCTB are largely retained even after denosumab therapy albeit significant reduction in osteoclast-like giant cells. In addition, the finding of positive staining within nuclei of cells both within and those rimming the newly formed osteoid/woven bone points toward the latter being laid down by neoplastic stromal cells of GCTB that have terminally differentiated toward osteoblastic phenotype, subsequent to blocking of inhibitory influence of RANK-RANKL interaction by denosumab. Lastly, the study also suggests that prior decalcification process may possibly affect IHC staining with antihistone antibodies.

\section{Authors' Contribution}

Anvesh Kamble and Monalisa Hui analyzed data, prepared manuscript, and did literature search. K. Nageshwara Rao and N. Ramakrishna reviewed the manuscript. $P$ Chandrasekhar was involved in manuscript review and supervision. Shantveer G Uppin was involved in conceptualization, definition of intellectual content, data analysis, manuscript review, and supervision. G Sadashivudu supervised the manuscript.

\section{Funding}

Nil.

\section{Conflict of Interest}

None declared.

\section{References}

1 Cowan RW, Singh G. Giant cell tumor of bone: a basic science perspective. Bone 2013;52(1):238-246

2 Wu PF, Tang JY, Li KH. RANK pathway in giant cell tumor of bone: pathogenesis and therapeutic aspects. Tumour Biol 2015;36(2):495-501

3 Yamagishi T, Kawashima H, Ogose A, et al. Receptor-activator of nuclear KappaB ligand expression as a new therapeutic target in primary bone tumors. PLoS One 2016;11(5):e0154680

4 Branstetter DG, Nelson SD, Manivel JC, et al. Denosumab induces tumor reduction and bone formation in patients with giant-cell tumor of bone. Clin Cancer Res 2012;18(16):4415-4424

5 Martin-Broto J, Cleeland CS, Glare PA, et al. Effects of denosumab on pain and analgesic use in giant cell tumor of bone: interim results from a phase II study. Acta Oncol 2014;53(9):1173-1179

6 Wojcik J, Rosenberg AE, Bredella MA, et al. Denosumab-treated giant cell tumor of bone exhibits morphologic overlap with malignant giant cell tumor of bone. Am J Surg Pathol 2016;40(1):72-80

7 Girolami I, Mancini I, Simoni A, et al. Denosumab treated giant cell tumour of bone: a morphological, immunohistochemical and molecular analysis of a series. J Clin Pathol 2016;69:240-247

8 Rekhi B, Verma V, Gulia A, et al. Clinicopathological features of a series of 27 cases of post-denosumab treated giant cell tumors of bones: a single institutional experience at a tertiary cancer referral centre, India. Pathol Oncol Res 2017;23(1):157-164 
9 Balke M, Hardes J. Denosumab: a breakthrough in treatment of giant-cell tumour of bone. ? Lancet Oncol 2010;11(3):218-219

10 Lipton A, Jacobs I. Denosumab: benefits of RANK ligand inhibition in cancer patients. Curr Opin Support Palliat Care 2011;5(3):258-264

11 Singh VA, Puri A. The current standing on the use of denosumab in giant cell tumour of the bone. J Orthop Surg (Hong Kong 2020;28(3):2309499020979750

12 Deveci MA, Paydaş S, Gönlüşen G, Özkan C, Biçer ÖS, Tekin M. Clinical and pathological results of denosumab treatment for giant cell tumors of bone: prospective study of 14 cases. Acta Orthop Traumatol Turc 2017;51(1):1-6

13 Roitman PD, Jauk F, Farfalli GL, Albergo JI, Aponte-Tinao LA. Denosumab-treated giant cell tumor of bone. Its histologic spectrum and potential diagnostic pitfalls. Hum Pathol 2017;63:89-97

14 Ud Din N, Umer M, Park YK. Histomorphometric analysis of pre- and post-denosumab-treated giant cell tumor of bone. Int J Surg Pathol 2020;28(8):859-867
15 Kato I, Furuya M, Matsuo K, Kawabata Y, Tanaka R, Ohashi K. Giant cell tumours of bone treated with denosumab: histological, immunohistochemical and H3F3A mutation analyses. Histopathology 2018;72(6):914-922

16 Wu CC, Hsieh PP. Denosumab-treated giant cell tumor of the bone mimicking low-grade central osteosarcoma. J Pathol Transl Med 2018;52(2):133-135

17 Behjati S, Tarpey PS, Presneau N, et al. Distinct H3F3A and H3F3B driver mutations define chondroblastoma and giant cell tumor of bone. Nat Genet 2013;45(12):1479-1482

18 Yamamoto H, Iwasaki T, Yamada Y, et al. Diagnostic utility of histone H3.3 G34W, G34R, and G34V mutant-specific antibodies for giant cell tumors of bone. Hum Pathol 2018;73:41-50

19 Lüke J, von Baer A, Schreiber J, et al. H3F3A mutation in giant cell tumour of the bone is detected by immunohistochemistry using a monoclonal antibody against the G34W mutated site of the histone H3.3 variant. Histopathology 2017;71(1):125-133 